\title{
Les collèges et le maintien du certificat
}

$\mathrm{P}$ eu de médecins ont passé leurs derniers examens de spécialité sans se dire avec soulagement, «ouf, c'est le dernier examen que j'aurai jamais à passer». Le soulagement pourrait cependant être de courte durée, car il se peut que d'autres examens se présentent : les deux collèges canadiens et nombre d'ordres provinciaux songent à durcir les normes de renouvellement de la licence et du certificat et à inclure dans ces mesures des examens ${ }^{1}$ (voir page 317 ).

L'évolution rapide de la recherche médicale impose aux médecins de consacrer de plus en plus de leur temps simplement à se garder à jour. Il leur a toujours fallu suivre les progrès de la science, mais les médecins doivent aussi composer maintenant avec l'élargissement du champ de pratique professionnel. Par exemple, selon la Charter on Medical Professionalism (charte du professionnalisme médical) ${ }^{2}$, le professionnalisme doit incorporer une évaluation continuelle de la qualité des soins et l'engagement envers le travail d'équipe afin de réduire les erreurs médicales et d'améliorer les résultats pour les patients. En même temps, les patients sont de mieux en mieux informés et ces utilisateurs sophistiqués des services de santé ne considèrent plus automatiquement les titres comme synonymes de connaissances et compétences. Ajoutez à l'équation une méfiance croissante envers le gouvernement et les autorités connexes (et envers les associations professionnelles lorsqu'elles poursuivent leurs propres intérêts). Résultat : une pression croissante sur les collèges pour qu'ils s'acquittent mieux d'évaluer la compétence de leurs membres.

Or, les collèges ont jusqu'ici limité leurs efforts surtout à documenter la participation à la formation médicale continue - faible prédicteur de compétence s'il en est. Il n'existe aucun examen officiel surveillé des connaissances et compétences équivalant à ceux qui sont maintenant exigés aux Etats-Unis pour le maintien du certificat ${ }^{3}$.

Cette lenteur est compréhensible. D'abord, l'étendue croissance de la pratique professionnelle complexifie davantage la tâche déjà ardue d'évaluer la compétence des médecins. Les évaluations, pour être valides et équitables, doivent être détaillées; elles doivent donc incorporer tout un éventail de critères qui sont en fait difficiles à mesurer, par exemple : un engagement envers l'apprentissage continu tout au long de la carrière, les compétences d'exercice en contexte pluridisciplinaire, la capacité à travailler avec succès dans les systèmes de soins et à les évaluer, et les compétences en communications interpersonnelles, éthique et professionnalisme.

Deuxièmement, on craint à juste titre que les outils actuels d'évaluation de la compétence soient peu fiables et d'une validité au plus marginale. Ces préoccupations sont justifiées jusqu'à un certain point, mais les outils d'évalua- tion s'améliorent. Il est vrai que les procédures d'examen modernes, surtout celles qui s'appliquent aux examens de réévaluation des connaissances et des compétences de gestion de cas en vue du maintien du certificat, ont amélioré leurs caractéristiques psychométriques et correspondent bien maintenant aux évaluations pratiques, du moins chez les stagiaires.

Troisièmement, des évaluations poussées périodiques coûtent cher. Les coûts entrent bien sûr en ligne de compte - comme le sait bien quiconque a déjà participé à un renouvellement de certificat aux États-Unis - mais ils sont à la baisse. De plus en plus, l'administration de ces évaluations et examens est décentralisée grâce à la vidéo par ordinateur, l'Internet ou autres moyens semblables. Les coûts continueront vraisemblablement à diminuer.

Les collèges devraient agir rapidement pour implanter des procédures plus rigoureuses de réévaluation et de renouvellement du certificat de leurs membres. Les ordres provinciaux ont une déjà une longueur d'avance. En $\mathrm{Al}$ berta, par exemple, les médecins sont évalués régulièrement par leurs patients, leurs pairs médecins, des experts-conseils, les médecins de référence et leur collègues de travail ${ }^{4}$. Des projets semblables sont en cours dans d'autres provinces.

Si les collèges ne font pas preuve d'un leadership dynamique en matière d'évaluation des médecins, de multiples formes (différentes et non transférables) d'examens et de procédures de renouvellement du certificat émergeront à l'échelle provinciale. Nous verrons aussi d'autres groupes d'agrément entrer en scène - tel le comité national américain d'assurance-qualité, qui a entrepris aux États-Unis d'agréer les médecins pour la gestion de certaines maladies comme le diabète ${ }^{5}$. Toute absence de progrès à ce chapitre aura pour effet d'accroître la méfiance du public et de réduire la valeur de la certification et, en permettant aux médecins de continuer à exercer sans avoir à démontrer qu'ils et elles répondent aux normes en vigueur, contribuera à de l'insatisfaction chez les patients et la population, à des événements indésirables et à des soins moins efficaces. - $7 A M C$

\section{Références}

1. Kondro W. Lifelong medical licences may end in 5 years. CMA7 2004; 171(4):317-8.

2. Medical professionalism in the new millennium: a physician charter. Ann Intern Med 2002;136(3):243-6. Disponible : www.annals.org/issues/v136n3 /full/200202050-00012.html (consulté le 22 juillet 2004).

3. Duffy FD, Zipes DP. The future of certification and recertification. Am $\mathcal{J}$ Med 2004;117:140-4.

4. Hall W, Violato C, Lewkonia R, Lockyer J, Fidler H, Toews J, et al. Assessment of physician performance in Alberta: the Physician Achievement Review. CMAf 1999;161(1):52-7.

5. National Committee for Quality Assurance. Diabetes physician recognition program. Disponible : www.ncqa.org/dprp/ (consulté le 23 juillet 2004). 